\section{THE TREATMENT OF WAR WOUNDS. ${ }^{1}$} II.

TREATMENT OF WOUND INFECTIONS.

I $F$ you have now quite clearly apprehended the sig1 nificant distinction between a live space and a dead space, you will with that have mastered the first great principle governing the treatment of all local bacterial infections. If you are dealing with infection in live spaces you can often mend matters by bringing (that is the rationale of hot fomentations) a larger blood supply-that means more lymph and more leucocytes - to the focus of infection; and again you can often mend matters by improving the quality of the lymph-that is the rationale of vaccine therapy ; or again, you may apply both these procedures concurrently. But when you are dealing with an infected dead space you cannot in these ways mend matters. You might just as well take a test-tubeful of infected fluid ind try by these means to influence it. Where you have infected dead spaces your remedial agent is the knife. You have to evacuate your dead space as I empty this test-tube.

Now that is the whole purpose and meaning of the surgery of the wound as carried out at the front. That can be summed up in two propositions. Every infected dead space must be cut down upon and evacuated. And, as a prophylactic measure, every space which would, if left to itself, become an infected dead space--that means every space occupied by an infected projectile or pieces of infected clothing or infected foreign bodies or devitalised infected tissues-must likewise be laid open and cleaned out. That exhausts the treatment of buried infections.

But it is only the beginning of the treatment of the wound. There is still the surface infection. The situation you have to face is just the same as that produced by emptying an infected tube; you have got rid of the infected contents, you have left the infection on the walls.

I now turn to the problem as to how best to deal with this infection. And here again inevitably we must establish distinctions. We must distinguish between the naked tissue surface made by the act of the projectile, or section with a knife, and the granulating defensive surface, which after a time clothes the nalked tissues. In the former we have a non-vascularised surface, and in this a system of lymph spaces left without mechanical or biological protection other than that fur nished by the emigration of leucocytes and (until that stanches) by the outflow of lymph. And the naked-tissue surface is not only ill-defended against microbic attack, it is also peculiarly iiable to damage and to physiological deterioration of the kind which opens the door wider to such attack. Such a surface readily dries up; and drying means the closing down of the capillary circulation. Again, a naked-tissue surface, seeing that it is non-vascularised, readily takes cold, and by that both lymph outflow and emigration are arrested. And, lastly, a naked-tissue surface, if kept. wet, will, so soon as the discharges become tryptic, readily undergo erosive digestion. Against all these forms of physiological degeneration specia! pro. vision should always be made.

A granulating surface offers much greater protection against microbic infection, and is much less subject to damage. The tissues are covered in by many layers of protective cells, the lymph spaces are sealed over, and there has been laid down immediately below the

1 By Sir Almroth E. Wright, C.B., F.R.S. In its original form this lecture was delivered at the Royal Institution on March 9. It was supplemented by additional matter relating to antiseptics and the method of Carrel, and was printed in full in the Lancet of June 23. Parts of the lectirre of purely technical interest have been omitted. Continued fron p. 518 .

NO. 2496, VOL. 99] surface in newly formed vessels a very abundant blcod supply. All this is protection against massive microbic invasion from the surface, against the wound taking cold, and against erosive digestion. In short, there is with an infected granulating surface much less danger of a set-back than with an infected naked-tissue surface.

\section{The Natural History of the Wound with a Naked- tissue Surface Left to Itself.}

Let us consider the natural history of the untreated wound with an infected naked-tissue surface. I will take the case of an open shell wound left without treatment. According as it is wet or dry the evolution of this wound will be entirely different. Let $x: s$ suppose that it is allowed to dry. Under the original dry dressing the blood and lymph flow from the surface will gradually stanch, and we shall then have a naked-tissue surface with a coating of coagulated bloof and lymph. In this will be incorporated elements of moribund tissue, other elements of foreign matter, anc always a certain number of microbes. Little by little the coating of coagulated blood and $1 \mathrm{ymph}$ upon the surface of the original wound, or of the surgeon's incisions, will dry up, and by that the capillary circulation will be closed down. And all the while the serophytic microbes will be proliferating. As a result of all this the superficial tissues will die and become gangrenous, and the originally clean naked-tissue surface will gradually be transformed into a diry, greenish-black, excessively foetid, slough-covered surfice pullulating with microbic srowth. ${ }^{2}$ Under the sloughs will then be formed infected dead spaces, and from these the infection-I am here thinking in particular of a gangrene infection-will invade the neigh bouring live spaces, converting these in their turn into dead spaces until we have to cope with large areas of gangrene and a general intoxication.

That, of course, will happen only with very heavy infection or extreme physiological deterioration. With lighter infection or less adverse physiological conditions the invaded organism will have recourse to measures of defence. Gradually the superficial sloughs and gangrenous portions of the deeper tissues will be demarcated and then amputated from the living tissues--the amputating agent being, no doubt, the tryptic ferment in the dead spaces. And at the same time there will have been organised in the living tissues some little way back a defensive front built up on the same plan as a granulating surface.

Let me now tell you also what will happen if the infected surface is simply lept wet. Here, also, the microbes which have been incorporated in the clot would grow out. Then there would supervene leucocytic emigration, and upon that would follow a breaking down of the leucocytes with a setting free of trypsin; and after that any and every microbe wouid pullulate in the cavity of the wound and on the devitalised wound-surface. Finally, if treatment were still deferred there would be reproduced in an aggravated form (for there would in the open wound be a varied and more formidable infection) the evil train of events which is associated with infection in a buried dead space. When you reflect that an open wound cavity filled with tryptic pus is physiologically equivalent to an unopened abscess sac, you will see that erosive action will enlarge and deepen its cavity; that this will enable the microbic infection to burrow everywhere deeper into the walls; and that bacterial poisons will be absorbed.

All I have been saying in the last few minutes can be

2 Let us note in connection with this that the albuminous substances of our tissues, when no longer bathed in lymph, are immediately degraded to
the rank of unprotected native albumens. 
compressed into this:-An infected naked-tissue surface becomes, if allowed to dry, a desiccated sloughcovered round; if simply kept wet, a tryptic suppurating wound. And the bacteriological events can also be expressed in a single sentence. A comparatively light infection, such as we have in the man whose wounds have been properly opened up and mechanically cleaned, is converted into a very heavy infection; and a purely surface infection into an infection invading the deeper tissues.

Problem of Preventing the Degeneration of the Wound When Treatment is Interrupted DURing TRANSPORT.

Having realised what happens to the wound when untreated, we have to think out how to keep wounds -whether originally completely open or opened by a surgeon-from falling during protracted journeys from hospital to hospital into these desperately unwholesome conditions. We have also to consider how to restore, as rapidly as possible, wounds which have lapsed into distressing conditions either through lying out untreated on the field or through interruption of treatment during lengthy transport.

Suggestion that the wound could be sterilised at the outset, and could be kept sierile by leaving an antiscptic in the wound.-The first thought of every man would probably be that the wound should be most carefully disinfected at the outset. But what happens in buins shows that to start in open wounds with is sterile surface avails nothing. A burn is at the outset absolutely sterile, and quite notoriously-no doubt the jerms begin to arrive before the burnt surface has weil cooled off-it tends to become very rapidly intractably septic. We may take it that the emigrating leucocites are hold back in the superficial sloughs, disintegrate there, and corrupt the exuding lymph. And this cannot be prevented by any application of antiseptics. It is just the same with war wounds. These become heavily infected even when they are drenched at the outset with the strongest antiseptics, such as undiluted carbolic acid and concentrated solutions of iodine.

This is not the place for any iengthy discussion of the reasons for this failure of antiseptics. But the gist of the matter can be put quite shortly. The current belief in the therapeutic efficicy of antiseptics rests on experiments which are quite fallacious. They are fallacious in that the antisentic in those experiments was applied in watery mediamedia which left that antiseptic unaffected. To have value-that is, to have application to conditions obtaining in vivo-the experiments should have been con. ducted in pus or serum-media which quench antiseptic action. Again, in the experiments of the past the antiseptics were intimately mixed with the bacterial suspensions; whereas, applied in the wound, the antiseptic comes only into external contact vith the infected wall and the inflowing discharges. Employed thus we cannot expect it to diffuse into and exert a bactericidal effect either in the infected wall or in the discharges.

By reason of these considerations having been disregarded, the issue as to whether antiseptics applied in the wound with prophylactic intent can be of any use must be investigated de novo.

\section{Experimental Investigation of the Efficacy of Anti: septics.}

Let me now try to indicate to you what sort of experiments should be undertaken before nourishing in connection with a particular antiseptic the expectation that it is going to be efficacious for sterilising and afterwards suppressing microbic growth in wounds. I can illustrate my points best if you let me show vou here four tubes.

In tube No. I I have a suspension of microbes in water. I now add an equal volume of the antiseptic I wish to test and shake up thoroughly. These are, as you see, conditions which give every possible advantage to the antiseptic. It is applied in a non-albuminous medium and is intimately mixed with the microbes. To find out whether the microbes have been killed I draw off a sample and dilute with very many times its volume of nutrient medium. I then incubate to see whether I get any bacterial growth.

In tube 2 I make the conditions more favourable to the survival of the microbes-infinitely more favourable than if I left behind an antiseptic in a wound. I have' here a mixture of staphylococci, streptococci, and gasgangrene bacilli suspended in serum, and I now, as in tube I, add an equal bulk of the antiseptic and shake up, and I then, following the technique of Prof. Beattie, pour on a little hot vaseline which will afterwards congeal. This, forming an air-tight seal, will allow the gangrene bacillus, if it survives, to grow out. It will also announce the growth of this microbe, for it will confine any gas which may be evolved from the culture.

Tube 3 is, as you see (Fig. Io), a tube which has

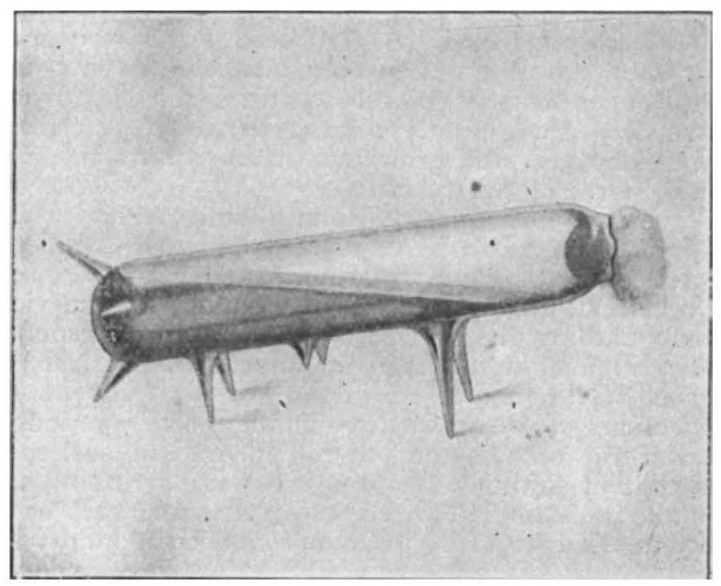
FIG. 10.-A test-tube standing on spike legs, representing a war

been drawn out into a number of hollow spikes to imitate the diverticula of the wound. My colleague, Dr. Alexander Fleming, its author and inventor, calls this form of tube the "artificial war wound." To imitate the conditions obtaining in the actual war wound we fill both the tube and its diverticula with an infected trypsinised serum. We now empty the tube, leaving behind of necessity in the diverticula a certain amount of the original infected fluid. We then fill with an antiseptic; and the future of the infection will now depend on the penetrating power of the antiseptic. If the antiseptic penetrates into the infected fluid sterilisation will be obtained; if it fails to penetrate, microbes will survive. To test our result we empty out the antiseptic, refill with trypsinised serum, and incubate.

After asking in tube 3 whether the antiseptic can completely sterilise a wound which has its recesses filled with an infected albuminous fluid, I go on in tube 4 to investigate the question as to how far the antiseptic can penetrate into the walls of the wound. Tube 4 is, as you see, a tube with hollow spikes. I have coated the inside with infected serum agar, and the spikes provide in their hollows a greater depth of 
infected lining and also securer purchase for the serum agar. The prepared tube is filled with antiseptic. And then we can, after an interval, pour out the antiseptic, fill in with nutrient broth or trypsinised serum, and then incubate. Any microbes which have been left alive in the lining will now grow out into colonies which can be inspected through the walls of the tube.

Let me show you a set of typical results obtained by the test-procodures just described, using Dakin's antiseptic, to-day perhaps the most popular of all antiseptics.

In tube $I$ we have obtained, as you can see by these subcultures, complete sterilisation. And it was obtained after only momentary contact with the antiseptic. In tube 2 , where a lightly infected serum was shaken up with an equal bulk of the antiseptic and then incubated, we have in our mixture of serum and antiseptic a very vigorous growth of microbes. You see the medium has become turbid, and there has been an evolution of gas which has pushed up the plug of congealed vaseline. In tube 3-and here the antiseptic stood for four hours in the tube-we have in the barrel a teeming multitude of microbes. And in tube 4, after four hours' contact with the antiseptic, only that very thin layer of the infected lining which coats the barrel has been sterilised, in the depth of every spike the bacterial colonies have come up quite thickly, and only in immediate contact with the antiseptic have the microbes been killed. And I here show you in a companion tube which has been incubated twenty-four hours longer that the microbes you have seen growing in the deeper layers very soo $x$ penetrate the sterilised superficial layer, and grow out in the culture medium in the barrel of the tube.

When we find an antiseptic giving results quite different from those here displayed it will then, for the first time, become a rational policy to use, and leave behind, an antiseptic in a wound with the view of safeguarding the patient during lengthy transport.

Suggestion that the bacterial infection in the wound can be kept down during transport by frequent reapplications of an antiseptic.-In the earlier period of the war the only method of re-applying an antiseptic was that of taking down the dressing, syringing the wound, and completely re-dressing. That was, especially in the case of deep wounds and compound fractures, a very lengthy and painful procedure, and one which was nearly impracticable in transport. For that complete re-dressing there has now been substituted by Carrel a procedure for washing and refreshing the surface of the wound through rubber tubes. According to Carrel, Dakin's antiseptic should be employed, and this should be applied every two hours. About the application of this in transport let me say this : that it would, I think, be impracticable to carry it out on a sufficiently large scale and sufficiently systematically; and Dakin's antiseptic applied in an unsystematic manner gives exactly the same results as simply keeping the wound wet.

Suggestion that the set-back in the wound during transport could be prevented by dressing with hypertonic salt solution.-The set-back in the wound with its resulting tragedies could, I think, be avoided by drawing out lymph in a continuous manner from the tissues, and holding up the emigration of leucocytes. The outflow of lymph would drive back and expel invading microbes. It would also prevent the conditions in the walls of the wound becoming unwholesome to leucocytes. The continuous outpouring of lymph would also effectively combat the corruption of the discharges in the cavity of the wound. And, lastly, it would prevent any drying up of the wound. The effect of holding up the emigration of leucocytes would be to prevent the corruption of the wound discharges.
You will remember that leucocytes, breaking down, furnish the trypsin which corrupts the discharges.

We have in a hypertonic solution the therapeutic agent we require for these purposes. The proper way of using it is to apply to the wound three or four layers of lint thoroughly soaked in 5 per cent. salt solution; to impose upon these, as a reinforcement, three or four more layers of lint thoroughly soaked in saturated salt solution, ${ }^{3}$ and then cover the whole with jaconet, or other impermeable material.

\section{Remedial. Treatment,}

I now pass from discussion of the method of preventing the set-back that occurs in transport to the discussion of its remedial treatment. The set-back will, as we have seen, have given us either a tryptic suppurating wound or a dry slough-covered wound. In each case the first item in treatment will be to get a clean surface. For that it will, in the case of the tryptic suppurating wound, suffice to wash away the tryptic pus. In the case of the desiccated sloughcovered wound we must get rid of the sloughs. The rational way to do that will be by cleansing digestion. Such cleansing digestion can be obtained by treating the wound with hypertonic salt solution. 'This will, as we have already seen, break down leucocytes, setting free trypsin, and then the free trypsin will rapidly, and especially rapidly if we let the hypertonic salt undergo dilution, amputate the dead from the living tissues. Let us note that what we set out to do by the use of hypertonic salt solution is only to achieve more rapidly, and, as we shall see, with less risk of infection, what putrefaction and the destruction of leucocytes by microbes would, if we allowed things to run their course, spontaneously effect. The second item of treatment in each case will be to combat the infection which has found a lodgment in the walls of the wound cavity. To deal with this we require an outpouring lymph stream, obtained by hypertonic salt solution.

If the train of reasoning I have laid before you is correct, it will follow that hypertonic salt solution is the agent we require both for preventing the set-back due to interruption of treatment in transport, and also for remedial treatment.

\section{Experiments which Exhibit the Properties of Hypertonic Salt Solution.}

You will very reasonably here expect me to produce experiments to show that a hypertonic salt solution has the virtues I ascribe to it. You will want to see for yourselves that it attracts water, draws out fluid from moist tissues, sets free trypsin from pus, and initiates digestion. There is room here ${ }^{4}$ only for the two following experiments, which have reference to the digestive cleansing of the wound.

Experiment I.-I have here, as you see, two testtubes filled nearly to the top with egg-albumen. To this was added $\frac{1}{2}$ per cent. of carbolic acid, and the albumen was then solidified by immersing the tubes in boiling water. That done, I took two cotton-wool plugs and steeped them in a pus to which I had added $\frac{1}{2}$ per cent. of carbolic acid. I then inverted my tubes, the one into a beaker containing 5 per cent. salt, the other into a beaker containing physiological salt solution. (Fig. II, A and B.) To these also I added $\frac{1}{2}$ per cent. of carbolic acid. You will understand why I chose carbolic acid as my antiseptic when I tell you that it is one which does not destroy trypsin or impede digestion. You see in the drawings made

3 The saturated solution diluted with six parts of water will give us ou per cent. sa't.

The exreriments on the drawing action of strong salt solution were set out in the fuller report of the lecture published in the Lancet of June 23 .

NO. 2496, VOL. 99] 
after the tube had been incubated for forty-eight hours, that in tube A, the tube which was immersed in hypertonic salt solution, the egg-albumen was extensively digested, while in tube $B$ there was only a mere trace of digestion.

Experiment 2.-I here try to imitate the conditions of slough-covered wounds. I have in these beakers a
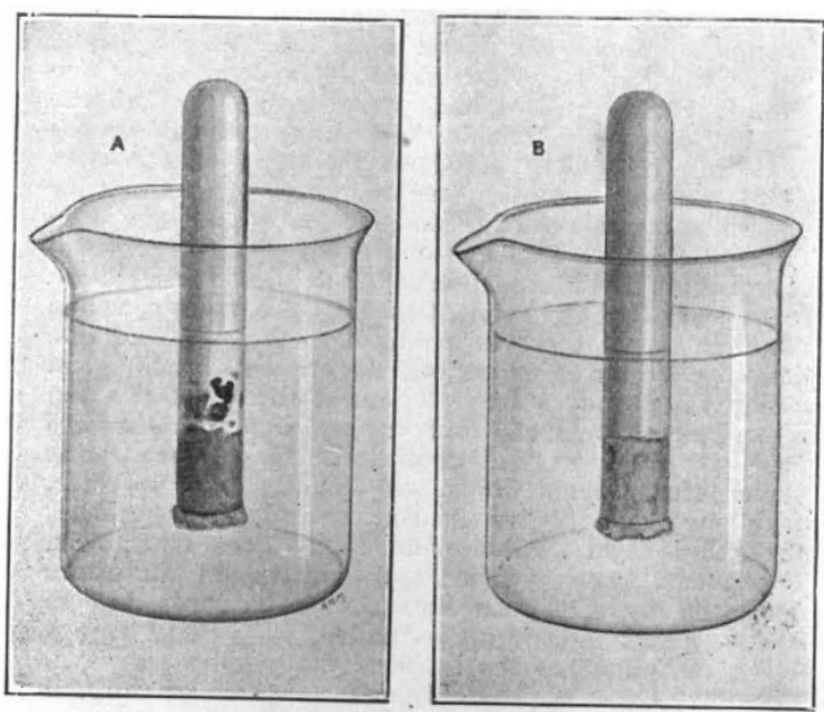

F1G. Ir.-Test-tubes filled with coagulated egg-albumen; then plugged with cotton-wool impregnated with pus; and then inverted into beakers. Beaker A contains hypertonic, beaker B normal salt solution.

foundation of coagulated white of egg containing 0.5 per cent. of carbolic acid. On the top of this I have in each case a disc of lint, woolly side up, firmly fastened down by adding another layer of egg-aibumen and coagulating this by heat. Upon the lint I have poured a non-tryptic pus, giving, of course, an equal amount to each beaker. In this way I have made what I think can pass as a fairly close representation

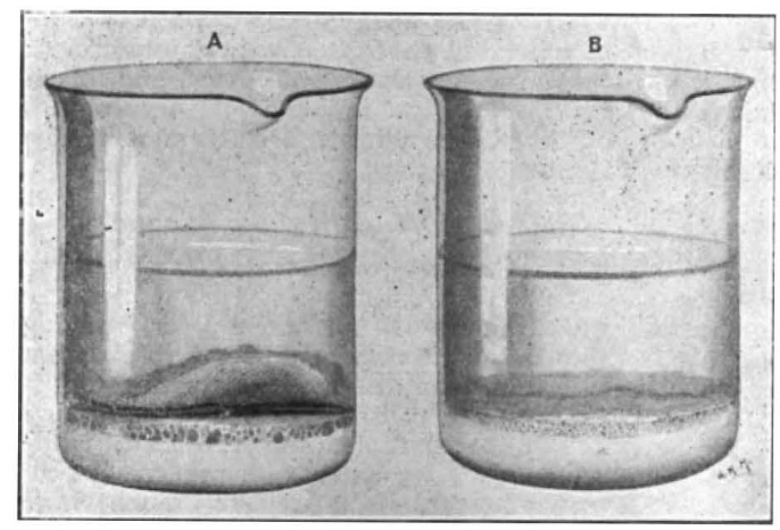

FIG. 12.-Beakers containing coagulated egg-albumen into which is embedded a layer of lint. Upon the lint was poured pus, and upon this in the case In beaker $\mathrm{A}$ the artificial slough has separated off by tryptic digestion.

of a pus-impregnated slough firmly adherent to the floor of a wound. (Fig. 12, A and B.)

We now pour upon one of the artificial sloughs 5 per cent.; upon another 0.85 per cent. solution made up with $\frac{1}{2}$ per cent. of carbolic acid; and we may pour upon a third Dakin's solution. We now place them all in the incubator. You see here what has happened after twenty-four hours. In beaker $\mathrm{A}$, where the artificial slough has been treated with hypertonic salt solution, the slough has loosened itself from its bed, and floats up as I pour in water. In beaker B, where I imposed only physiological salt solution, the slough is still firmly adherent. And the same holds of beaker C (not figured), where we have Dakin's solution.

Treatment of the Wound in the Case Where we HAVE ONLY a SURFACE INFECTION.

When we have got back to a clean and only lightly infected surface we must think out our next step. It will help if we first review what we have learned and get things into proper perspective.

We have learned that there are in wound infections two supreme dangers. First, there is the danger associated with the buried infection. We have appreciated that the effective and only remedy for this is the immediate opening up of the infected dead spaces. That, you will remember, is a question of converting a buried infection into a surface infection. The second very serious danger is that intensification of the surface infection which follows upon a lengthy interruption of treatment during transport. This, regarded from the point of view of loss of life and limb, ranks next in order of importance after delay in dealing with the buried infection. When the set-back due to transport has been prevented or remedied, we have confronting us the problem which, if treatment had been uninterrupted, would have presented itself earlier-the problem as to how to treat a slight infection of a naked-tissue surface.

One procedure is to leave the wound to heal up from the bottom, limiting oneself to such re-dressing as would prevent erosive digestion. By this programme the patient would, when his wound is a large one, be condemned to very many months of disability and also of bacterial intoxication. For the fact has got to be faced that it is all but impossible to maintain satisfactory conditions in a large wound for months on end.

The alternative programme is for the surgeon to close the wound with the minimum delay. If the anatomical conditions permit, and the bacteriological examination shows the wound surface to be practically uninfected, or if the wound is only a very few hours old and the implanted microbes cannot yet have grown out, the wound can, after removal of all dead and foreign matter, be immediately closed-the surgeon, of course, standing by to reopen the wound if symptoms of buried infection develop. If, on the other hand, bacteriological examination shows that the wound surface is appreciably infected, or the history of the case makes this practically certain, we should, by closing the wound, be violating all the principles of surgery. We should be converting a surface infection into a buried infection. The proper step to take with a wound which is appreciably infected is to reduce the microbic infection to the point at which it is negligible and then re-suture.

\section{Methods of Dealing with a Microbic Infection which} Stands in the Way of Secondary Suture.

The microbic infection may be dealt with by any one of the following procedures.

In the first place we can employ the physiological procedure. If we elect to do this, we must think out clearly the requirements. For example, it will be inappropriate when dealing with a purely superficial streptococcic and staphylococcic infection to continue the application of hypertonic salt solution. The effect of that would be, on one hand, to hold off phagocytes from the microbes (for strong salt arrests

No. 2496, VOL. 99], 
emigration); and, on the other, to provide the staphylococcus and streptococcus with lymph, a fluid in which they can grow and disseminate themselves over the whole face of the wound. What we want is an application which calls out leucocytes, which will restrain, or at any rate will not activate, the lymph flow. Physiological salt solution, and zinc sulphate in $\frac{1}{4}$ per cent. solution, and no doubt many other heavy metal salts in dilute solution, are the sort of agents we require. But what is, above all, essential to success in physiological treatment of a surface infection is assiduity in removing any leucocytes which may break down upon the face of the wound. That is a question of maintaining intact the antitryptic power of the lymph on the wound surface.

A second method of procedure-I may call it the unreasoning antiseptic procedure-is to employ an antiseptic, without laying stress upon the assiduous cleansing of the wound surface and the maintenance of good physiological conditions; without inquiring whether the antiseptic can, when brought into external contact with pus or an infected tissue, penetrate into it; and without asking whether the antiseptic hinders phagocytosis, or destroys the antitryptic power of the blood fluids, or permits or interferes with tryptic action.

This unreasoning antiseptic procedure is constantly employed. It has led to failure upon failure, and it would be a matter for wonder if it did succeed.

The third and last method of procedure 1 may call the combined antiseptic and physiological procedure. If we want to find a method of this sort we shall not find it by inquiring for it under this name. What we have to seek is a method which proclaims itself an antiseptic method and in this guise combats effectively, but perhaps not with full comprehension, corruptive changes in the wound.

The method of Carrel is, as I think, such a method. I would propose to show that it is a combined antiseptic and physiological method; then to survev the results obtained; and, finally, to consider how far the results should be credited to the antiseptic, and how far to the physiological, element in the treatment.

We have in Carrel's treatment two factors: (a) Dakin's antiseptic, or, as I should prefer to call it, Dakin's therapeutic agent; and (b) Carrel's procedure for washing and refreshing the wound surface in the intervals between the complete dressings. Now each of these factors acts not only by killing or removing microbes, but also by making the conditions in the wound unfavourable for microbial growth. Lei me, taking first Dakin's fluid and then Carrel's washing procedure, try to make for you an inventory of their directly anti-bacterial, and their physiological or indirectly anti-bacterial, effects.

\section{Dakin's Fluid.}

Dakin's fluid is, as I have shown you, ${ }^{5}$ a very ineffective antiseptic when it is brought into application upon microbes suspended in serum. It is also, as I have likewise shown you, an antiseptic which has as good as no power of penetrating into alluminous fluids. It is also an extremely volatile antiseptic. When exposed in a shallow dish at blood temperature I have found it to lose four-fifths of its potency in half an hour, and it will, as I have already had occasion to point out, if not already quenched by contact with serum, very quickly disappear from the wound.

Turning from the effect exerted cpon microbes to the effect exerted upon the wound surface, let me recall to those of you who have seen it that when a naked-tissure surface is treated with Dakin's fluid (or for the matter of that, with 5 per cent. salt solution)

$$
5 \text { Viale supra, Experiments on Antiseptics. }
$$

No. 2496, VOL. 99] it is speedily converted into a bright coral-red granulating surface. That means it is converted into a defensive surface excellently well provided with newformed blood-vessels from which active leucocytes and fully potent lymph will emerge. 'I hat is a physiological action to the good. But there are also other effects exerted. Leucocytes are affected by Dakin's fluid. Experiments show that it is destructive to phagocytosis. When we add one part of the reagent to nine of excoagular blood we reduce the phagocytic power of that blood by more than one-half. ive abolish phagocytosis when we add one part of the reagent to four of blood. The fluid elements of the discharge also are altered in character by Dakin's fluid. Let me remind you here that we saw in our experiments on artificial sloughs that treatment with Dakin's solution hinders the digestive processes which bring about their separation. This stands in relation to the fact that the reagent exerts upon trypsin, when albumen is not there to act as a buffer, a destructive action. We have, as you perceive, here a physiological action which may quite well come into operation when a comparativcly clean but tryptic wound surface is flushed. Dakin's fluid abolishes also the antitryptic power of the blood fluids. It would seem, therefore, with one hand to give protection, and with the other to take it away. But what really does happen is, I suppose, that trypsin and antitrypsin alike are destroyed by the flush and that afterwards in the wound a new beginning is made.

Let us follow up the train of thought here started. We may, I think, profitably ask ourselves whether, if put to our election between mairtaining antiseptic action continuously at the expense of physiological action, and alternating antiseptic with physiological action, we should not do well to elect for the latter policy. And we may muse whether it was not specially felicitous to have employed, as Carrel has done, an antiseptic which is very readily quenched and also very volatile and to have applied it discontinuously. Had that antiseptic been employed by a method of continuous irrigation, phagocytosis on the face of the wound would have been excluded, and we might have had in the cavity of the wound a lymph the antitryptic power of which had been destroyed.

But I have already said enough about Dakin's fluid if you have appreciated that it is a poor antiseptic; that it acts as a poison upon leucocytes and blood fluids; that its physiological action is a very complicated one; and that its beneficial effects cannot be due simply to its antiseptic action.

\section{Carrel's Method of Irrigating the Wound.}

I now come t; Carrel's procedure of intercalating between the complete dressings a frequent flushing and refreshing of the wound surface and for carrying out this flushing unlaboriously. Allow me to say that we have here, I think, far the most important contribution made to surgical technique since the beginning of the war. But to that let me add that, while Carrel's procedure gives us a new and improved technique for the application of antiseptics, much more does it give us a new and improved technique for physiological treatment. In all physiological treatment the assiduous removal of corrupted and corruptible discharges is the primary desideratum.

We now turn to the results of the treatment of infected wound surfaces by Carrel's method, and we mav take them from Carrel's book. But it will be well, in order to keep to the kind of wound infection here under discussion, to exclude from consideration wounds complicated with fractures-for in those effective washing is difficult. And we may further, looking to the classification of wounds of soft parts in 
Carrel's book, exclude from consideration his class of phlegmonous and gangrenous wounds and his class of suppurating wounds. These would correspond to wounds which have, through postponement of treatment or its interruption by transport, suffered a setbatck, converting an originally light surface infection into a heavy infection with invasion of the deeper tissues. There would then fall within our purview only his class of fresh wounds of soft parts taken in hand when five to twenty-four hours old. And we learn from the data he gives with respect to these that, where there are sloughs, fifteen to twenty days, and, where there are none, five to twelve days, are required to prepare the wound for secondary suture. That gives us a measure of what can be done by what I have, I hope not unjustifiably, called Carrel's "combined antiseptic and physiological treatment.'

Let us consider what Carrei's results tell us. They tell us in the first place that, whatever else it is, Carrel's treatment is not in any sense a therapia magna sterilisans. Regarded as an antiseptic method, it is a method of "fractional steriitisation" requiring lor the case we are considering-the simplest case of all-at the rate of twelve douches a day a series of 60 to 144 antiseptic douches. And if I am right in regarding Carrel's treatment as a combined antiseptic and physiological treatment, we have, superadded to the antiseptic, a series of 60 to I44 physiological attacks upon the microbes- each such attack starting from an atryptic condition.

The consideration of these figures leads directly to what I have to say in conclusion. While Carrel's work constitutes a very notable practical achievement, resarded as science it comes short in the respect that adequate control experiments are lacking. I do not mean that it has not been demonstrated that Carrel's treatment accomplishes what was impossible by the old system of syringing with antiseptics and leaving the wound afterwards to fill with pus. The inefficacy of that older treatment was attested by tens and hundreds of thousands of control experiments. What I mean is that we have not in Carrel's work any control experiments with more potent and penetrating antiseptics to negative the idea that with these one could with fewer than 60 to 144 consecutive douches convert a light surface infection into a negligible one. And again, we have not from Carrel any control experiments with a well-thought-out physiological treatment to negative the idea that one could achieve a similar sterilisation by 60 to 144 successive physiological attacks upon the microbes, starting each time from an atryptic condition.

If we would abide in the spirit of science, every unwarranted assumption must go. We must not assume that when we have successfully combated a surface infection by a series of 60 to 144 therapeutic operations we have reached finality. And much less must we, from the fact that a treatment successfully combats surface infections, infer that it is also an effective treatment for infections which penetrate into the deeper tissues. It ought to come home to us instead that it is impossible that for quite different categories of wounds, i.e. for quite diverse conditions, there should be any one routine treatment.

\section{UNIVERSITY AND EDUCATIONAL INTELLIGENCE.}

A PAMPHLET has been received from University College, London, giving full particulars of the University centre for preliminary and intermediate medical studies arranged in connection with the faculty of medical sciences of the college. The college faculty of medical sciences comprises the departments of physics, chemistry, botany, and zoology (the preliminary medical sciences), also the departments of anatomy, physiology, and pharmacology (the intermediate medical sciences), and the departments of hygiene and public health and of pathological chemistry (post-graduate study). Each of the departments is also equipped for more advanced work and provides facilities for research. Numerous scholarships and exhibitions are available for intending medical students, detailed regulations concerning which can be obtained on application to the secretary, Dr. W. W. Seton, at the college.

THE calendar for the session $1917-18$ of the Royal College of Science for Ireland has now been published. The college provides a complete course of instruction in those branches of science which are connected with agriculture, engineering, and manufactures, and it trains teachers of science for technical and secondary schools. By the prosecution of researches in pure and applied science the college has been able to render aid to the agricultural and industrial development of Ireland. The regular courses of study extend over four years, and lead to the associateship of the college. The fellowship of the college may be awarded to any associate of at least three years' standing who submits a thesis, which shall meet with the approval of the dean and council, embodying the results of his own original scientific research, or who has submitted satisfactory evidence that he has contributed in a marked degree to the advancement of science. A limited number of research studentships may be awarded each session to qualified persons who desire to prosecute approved lines of research. The college is administered by the Department of Agriculture and Technical Instruction for Ireland.

\section{SOCIETIES AND ACADEMIES PARIS.}

Academy of Sciences, August 13.-M. Paul Appell in the chair.-G. Humbert: The reduction $(\bmod 2)$ of quadratic binary forms.-C. Richet and H. Cardot : A new method of determining the reducing substances in urine. The diluted urine is allowed to act upon an acidified solution of potassium permanganate under conditions such that the urea is not oxidised. A manganese coefficient for normal urine is established, and this is shown to be independent of the total urea excreted.-E. Cahen: The series of best absolute approximation for a number.--L. Picart : The total eclipse of the moon of July 4, 1917. Observations made at the Bordeaux-Floirac Observatory showed that during the whole period of total eclipse the north edge of the moon was more luminous than the south edge; the western edge was more luminous up to the middle of the eclipse.-G. Sizes: The German gamma termed "harmonic," or "exact," or improperly modern, from the point of view of musical acoustics.--P. Portier : The physiological rôle of symbiotic micro-organisms.MM. Abelous and Aloy : The biochemical phenomena of. oxido-reduction. Repeating the experiments of Bach on the ferment in milk, it was found that a large number of substances besides aldehydes may act as co-ferments, such as amines, terpenes, and manganese salts. Details are given of the simultaneous reduction of sodium chlorate and oxidation of salicylic aldehyde. -Mme. C. Cardot and H. Cardot: The analogy between the lactic ferments and streptococci from the point of view of the action of disinfectants. The growths of the lactic bacillus and streptococcus under the action of increasing amounts of two antiseptics, sodium fluoride and phenol, were compared. The curves expressing the results of the experiments show close agreement, and the authors conclude that laws 